Pacific Journal of Mathematic 


\title{
QUASI-CONVEXITY AND THE LOWER SEMICONTINUITY OF MULTIPLE INTEGRALS
}

\author{
Charles B. Morrey, Jr.
}

1. Introduction. We are concerned in this paper with integrals of the form

$$
I(z, D)=\int_{D} f\left[x, z^{i}(x), z_{x^{a}}^{i}(x)\right] d x,
$$

where

$$
\begin{aligned}
x=\left(x^{1}, \cdots, x^{\nu}\right), \quad z=\left(z^{1}, \cdots,\right. & \left.z^{N}\right), p=p_{\alpha}^{i} \\
& (i=1, \cdots, N ; \alpha=1, \cdots, \nu),
\end{aligned}
$$

$f(x, z, p)$ is continuous in its arguments, and $D$ is a bounded domain.

The object of the paper is to discuss necessary and sufficient conditions on the function $f$ for the integral $I$ to be lower semicontinuous with respect to various types of convergence of the vector functions $z$. Because of the success of the "direct methods" in the Calculus of Variations, many writers have shown that certain integrals are lower semicontinuous. However, the writer knows of no paper in which a necessary condition for lower semicontinuity was discussed, although such a condition is very easy to obtain (see Theorem 2.1).

In $\$ 2$, a general condition called "quasi-convexity" (see Definition 2.2) on the behavior of $f$ as a function of $p$ is obtained which is both necessary and sufficient for the lower semicontinuity of $I$ with respect to the type of convergence given in Definition 2.1. This condition is that any linear function furnish the absolute minimum to $I(z, D)$ among all Lipschitzian (see below) functions which coincide with it on $D^{*}, D$ being any bounded domain and $D^{*}$ its boundary; here, of course, we consider $f$ to be a function of $p$ only. Section 3 discusses cases involving more general types of convergence and gives an existence theorem. In $\$ 4$, it is shown that if $f(p)$ is continuous and quasi-convex, then it satisfies a certain generalized Weierstrass condition which reduces to the ordinary one (for the case at hand) when $f$ is of class $C^{\prime}$; this is, in turn, seen to be equivalent to the Legendre-Hadamard condition (see (4.8)) (quasi-regularity in its general form) when $f$ is of class $C$. In $\$ 5$, a general sufficient

presented to the American Mathematical Society at the Summer meeting of 1949 in Boulder, Colorado, under the title "Quasi-convexity and the lower semicontinuity of double integrals".

Received February 19, 1951.

Pacific J. Math. 2 (1952), $25-53$ 
condition for quasi-convexity is proved and the necessary condition of $\S_{4}$ is seen to be sufficient when $f$ is either a quadratic form in the $p_{\alpha}^{i}$ or is the integrand of a parametric problem with $N=\nu+1$. The view of Terpstra's negative result [5] that even the strong Legendre-Hadamard condition $(>0)$ does not necessarily imply the existence of an alternating form $C_{i j}^{\alpha \beta} p_{\alpha}^{i} p_{\beta}^{j}\left(C_{i j}^{\beta \alpha}=-C_{i j}^{\alpha \beta}\right.$, and so on) such that

$$
f(p)+C_{i j}^{\alpha \beta} p_{\alpha}^{i} p_{\beta}^{j} \equiv\left(a_{i j}^{\alpha \beta}+C_{i j}^{\alpha \beta}\right) p_{\alpha}^{i} p_{\beta}^{j}
$$

is positive definite when $\nu>2$, it would seem that there is still a wide gap in the general case between the necessary and sufficient conditions for quasiconvexity which the writer has obtained. In fact, after a great deal of experimentation, the writer is inclined to think that there is no condition of the type discussed, which involves $f$ and only a finite number of its derivatives, and which is both necessary and sufficient for quasi-convexity in the general case.

In (1.2), we have used the usual tensor summation convention, and will continue to use it throughout the paper; unless otherwise specified, the Greek letters will run from 1 to $\nu$ and the Latin letters from 1 to $N$.

We shall denote the sum and difference of vectors of the various sorts $(x, z$, $p$, and so on) in the usual way. We shall define

$$
|x|=\left(x^{\alpha} x^{\alpha}\right)^{1 / 2}, \quad|z|=\left(z^{i} z^{i}\right)^{1 / 2},|p|=\left(p_{\alpha}^{i} p_{\alpha}^{i}\right)^{1 / 2} .
$$

If $\zeta(x)$ is a vector function with derivatives, $\pi(x)$ will denote the vector function $\pi_{a}^{i}(x)=\zeta_{x a}^{i}(x)$; similar notations involving other letters will be introduced as the occasion demands.

All integrals are Lebesgue integrals, frequently of vector functions. It is sometimes desirable to consider the behavior of a function $z(x)$ with respect to a particular variable $x^{\alpha}$ or to the $\nu-1$ variables $\left(x^{1}, \cdots, x^{a-1}, x^{\alpha+1}\right.$, $\left.\cdots, x^{\nu}\right)$. In such a case, we write $x_{\alpha}^{\prime}$ for $\left(x^{1}, \cdots, x^{\alpha-1}, x^{\alpha+1}, \cdots, x^{\nu}\right)$, $\left(x_{\alpha}^{\prime}, x^{\alpha}\right)$ for $x$ and so on. It is also convenient to write the boundary integrals

$$
\int_{D^{*}} A_{\alpha}(x) d x_{\alpha}^{\prime},
$$

where each $A_{\alpha}(x)$ may be a vector $A_{\alpha}^{i}(x)$ and the boundary $D^{*}$ of the domain is sufficiently regular; such an integral is to be regarded as a Lebesgue-Stieltjes integral with respect to the set function $x_{\alpha}^{\prime}(e)$ on $D^{*}$ chosen so that Green's theorem

$$
\int_{D^{*}} \zeta^{i} d x_{\alpha}^{\prime}=\int_{D} \zeta_{x \alpha}^{i} d x
$$


holds. The closure of a set $E$ will be denoted by $\bar{E}$.

Ordinary functions of class $\Re_{s}$, $\Re_{s}^{\prime}$, 被”, and so on, $s \geq 1$, have been discussed at length in the papers [1] and [2]; the extension to vector functions is trivial. We define the integrals $\bar{D}_{s}(z, G)$ and $D_{s}(z, G)$ by

$$
\bar{D}_{s}(z, G)=\int_{G}|z(x)|^{s} d x+L_{s}(z, G), D_{s}(z, G)=\int_{G}\left[z_{x \alpha}^{i}(x) z_{x a}^{i}(x)\right]^{s / 2} d x
$$

Each function $z$ of class $\Re_{s}$ is equivalent to a function $\bar{z}$ defined uniquely almost everywhere as that number such that the Lebesgue derivative of the set function

$$
\int_{e}\left|z(x)-\bar{z}\left(x_{0}\right)\right|^{s} d x
$$

is zero at $x_{0} ; \bar{z}$ is supposed to be defined at every point $x_{0}$ where such a number exists; $\bar{z}$ is of class $\Re_{s}^{\prime}$ (see [1] and [2]) and is also of class $\Re_{s}^{\prime}$ in any coordinate system related to the original by a regular Lipschitzian transformation (cf. [2], Theorem 6.3; the $\bar{z}$ there used has a slightly different definition from the present one but the present theorem has been proved for vectors $z$ with values in a Riemannian manifold in [4], Lemma 2.3 and Theorem 2.5).

A function $z$ is said to satisfy a (uniform) Lipschitz condition with coefficient $M$ on a set $S$ if and only if

$$
\left|z\left(x_{1}\right)-z\left(x_{2}\right)\right| \leqq M\left|x_{1}-x_{2}\right|, x_{1} \in S, x_{2} \in S .
$$

A function is Lipschitzian if it satisfies a Lipschitz condition.

If $g(y), y=\left(y^{1}, \ldots, y^{n}\right)$, is summable on a domain $D$, we define the $h$ average function $g_{h}$ by

$$
g_{h}(y)=(2 h)^{-n} \int_{y-h}^{y+h} g(\eta) d \eta, \quad h>0 ;
$$

if $g$ is summable then $g_{h}$ is continuous where defined; if $g$ is continuous on $D$ then $g_{h}$ is of class $C^{\prime}$ and $g_{h}$ tends uniformly to $g$ on each bounded closed set interior to $D$; if $g$ is of class $\Re_{s}$ on $D$ then $g_{h}$ tends strongly in $\beta_{s}$ to $g$ on each domain $G$ with $\bar{G} \subset D$ (see [1], Lemma 5.1).

A form

$$
C_{i_{1}, \cdots, i_{\mu}}^{a_{1}, \cdots, a_{\mu}} \pi_{a_{1}}^{i_{1}} \ldots \pi_{\alpha_{\mu}}^{i_{\mu}}\left(\mu \leqq \nu, 1 \leqq \alpha_{\gamma} \leqq \nu, 1 \leqq i_{\gamma} \leqq N, y=1, \cdots, \mu\right),
$$

is called alternating if and only if the $C$ 's satisfy the obvious symmetry requirements and also the antisymmetry condition that 


$$
C_{i_{1}, \cdots, i_{\mu}}^{\beta_{1}, \cdots, \beta_{\mu}}= \pm C_{i_{1}, \cdots, i_{\mu}}^{\alpha_{1}, \cdots, \alpha_{\mu}}
$$

according as $\left(\beta_{1}, \cdots, \beta_{\mu}\right)$ is an even or odd permutation of the indices $\left(\alpha_{1}, \ldots, \alpha_{\mu}\right)$; if $\zeta(x)$ is a vector function, then

$$
\mu ! C_{i_{1}, \cdots, i_{\mu}}^{a_{1}, \cdots, a_{\mu}} \pi_{a_{1}}^{i_{1}}(x) \cdots \pi_{\alpha_{\mu}}^{i_{\mu}}(x)=C_{i_{1}}^{\alpha_{1}}, \ldots, i_{\mu} \frac{\partial\left(\zeta^{i_{1}}, \ldots, \zeta^{i_{\mu}}\right)}{\partial\left(x^{a_{1}}, \ldots, x^{\alpha_{\mu}}\right)}
$$

the fractions on the right denoting Jacobians.

2. A necessary and sufficient condition for lower-semicontinuity. We begin with some definitions.

DEFINITION 2.1. For the purposes of this section, we say that the vector functions $z_{n}$ tend to the vector function $z$ on the domain $D$ if and only if the $z_{n}$ and $z$ all satisfy a uniform Lipschitz condition on $D$, independent of $n$, and the $z_{n}$ tend uniformly to $z$ on $D$. We shall write $z_{n} \rightarrow z$ to denote this type of convergence.

DEFINITION 2.2. A function $f\left(p_{a}^{i}\right)$ is said to be quasi-convex if and only if

$$
\int_{D} f[p+\pi(x)] d x \geqq f(p) \cdot m(D), \quad \pi_{\alpha}^{i}(x)=\zeta_{x \alpha}^{i}(x),
$$

for each constant $p$, each domain $D$, and each vector function $\zeta$ which satisfies a uniform Lipschitz condition on $D$ and vanishes on $D^{*}$.

We shall show in this section that the integral $I(z, D)$ is lower semicontinuous with respect to the type of convergence specified in Definition 2.1 on each bounded domain $D$ if and only if $f(x, z, p)$ is quasi-convex in $p$ for each fixed $(x, z)$.

THEOREM 2.1. Suppose $I(z, D)$ is lower semicontinuous with respect to the type of convergence indicated on every region $D$. Then $f$ is quasi-convex in $p$ for each fixed $(x, z)$.

Proof. Let $x_{0}$ be any point, $R$ be the cell $x_{0} \leqq x^{i} \leqq x_{0}^{i}+h, Q$ be the cell $0 \leqq x^{i} \leqq 1$, and $\zeta$ be any function of class $C^{\prime}$ and periodic in each $x^{i}$ with period 1. Let $z_{0}$ be any function of class $C^{\prime}$ on $R$.

For each $n$, define $\zeta_{n}(x)$ on $R$ by 


$$
\zeta_{n}^{i}(x)=n^{-1} h \zeta^{i}\left[n h^{-1}\left(x-x_{0}\right)\right]
$$

Then

$$
\zeta_{n x^{\alpha}}^{i}(x)=\zeta_{x^{\alpha}}^{i}\left[n h^{-1}\left(x-x_{0}\right)\right]
$$

and

$$
\begin{aligned}
I\left(z_{0}+\zeta_{n}, R\right) & =\int_{R} f\left\{x, z_{0}^{i}(x)+\zeta_{n}^{i}(x), p_{0 \gamma}^{i}(x)+\pi_{\gamma}^{i}\left[n h^{-1}\left(x-x_{0}\right)\right]\right\} d x \\
= & \sum_{a} \int_{R_{a}}\left(f\left\{x, z_{0}^{i}(x)+\zeta_{n}^{i}(x), p_{0 \gamma}^{i}(x)+\pi_{\gamma}^{i}\left[n h^{-1}\left(x-x_{0}\right)\right]\right\}\right. \\
& \left.-f\left\{x_{\alpha}, z_{0}^{i}\left(x_{\alpha}\right), p_{0 \gamma}^{i}\left(x_{a}\right)+\pi_{\gamma}^{i}\left[n h^{-1}\left(x-x_{0}\right)\right]\right\}\right) d x \\
+ & \sum_{a}\left(n^{-1} h\right)^{\nu} \int_{Q} f\left\{x_{a}, z_{0}^{i}\left(x_{\alpha}\right), p_{0 \gamma}^{i}\left(x_{\alpha}\right)+\pi_{\gamma}^{i}(\xi)\right\} d \xi,
\end{aligned}
$$

where

$$
\begin{aligned}
& \alpha=\left(\alpha_{1}, \ldots, \alpha_{\nu}\right), R_{\alpha}=R_{a_{1}}, \ldots, \alpha_{\nu}, n^{-1}\left(\alpha_{\beta}-1\right) \leqq x^{\beta} \leqq n^{-1} \alpha_{\beta}, \\
& x_{\alpha}=\left(x_{a_{1}}^{\beta}, \ldots, \alpha_{\nu}\right), x_{a_{1}}^{\beta}, \ldots, \alpha_{\nu}=n^{-1}\left(\alpha_{\beta}-1\right), \beta=1, \cdots, \nu .
\end{aligned}
$$

As $n \rightarrow \infty$, we see, since $f$ is uniformly continuous on any bounded part of space, $\zeta_{n}(x)$ tends uniformly to zero, and the $\pi_{\gamma}^{i}$ are bounded, that

$$
\lim _{n \rightarrow \infty} I\left(z_{0}+\zeta_{n}, \ddot{K}\right)=\int_{R}\left[\int_{Q} f\left[x, z_{0}(x), p_{0}(x)+\pi(\xi)\right] d \xi\right] d x
$$

From the lower semicontinuity of $I$, we must have

$$
\int_{R}\left\{\int_{Q} f\left[x, z_{0}(x), p_{0}(x)+\pi(\xi)\right] d \xi\right\} d x \geqq \int_{R} f\left[x, z_{0}(x), p_{0}(x)\right] d x .
$$

Now, let $x_{0}, z_{0}$, and $p_{0}$ be any constant vectors. By letting

$$
z_{0}(x)=z_{0}+p_{0 \alpha}\left(x^{\alpha}-x_{0}^{\alpha}\right)
$$

dividing by $h^{\nu}$ and letting $h \rightarrow 0$, we obtain

$$
\int_{Q} f\left[x_{0}, z_{0}, p_{0}+\pi(\xi)\right] d \xi \geqq f\left(x_{0}, z_{0}, p_{0}\right)
$$


By approximations, we can extend this to all $\zeta$ which satisfy a uniform Lipschitz condition over the whole space and are periodic of period 1 in each $x^{\alpha}$.

Now, let $D$ be a bounded doma in and suppose $\zeta$ satisfies a uniform Lipschitz condition on $D$ and vanishes on $D^{*}$. Let $R$ be a hypercube of edge $h$, with edges parallel to the axes which contains $D$. Extend $\zeta$ to the whole space by first defining it to be zero on $\bar{R}-D$ and then extending it to be periodic of period $h$ in each variable. Then a simple change of function and variable reduces $R$ to $Q$ and establishes the result.

L.emma 2.1. Suppose $R$ is a cell with edges $\left(2 h^{1}\right), \cdots,\left(2 h^{\nu}\right)$ and center $x_{0}$. Let $h$ be the smallest $h^{\alpha}$. Suppose also that $0<k<h$, that $\zeta^{*}$ satisfies a uniform Lipschitz condition with coefficient $M \geqq 1$ on $R^{*}$, and suppose

$$
\left|\zeta^{*}(x)\right| \leqq k, x \in R^{*}
$$

Then there is a function $\zeta$ on $\bar{R}$ which satisfies a Lipschitz condition with coefficient $M$ on $\bar{R}$, coincides with $\zeta^{*}$ on $R^{*}$, and is zero except on a set of measure at most

$$
m(R) \cdot\left[1-\left(1-h^{-1} k\right)^{\nu}\right]
$$

Proof. Let $R_{1}$ be the cell with center at $x_{0}$ and edges $2\left(h^{\alpha}-k\right), \alpha=1$, ..., $\nu$. Then, since $h=\min h^{a}$, we have

$$
m\left(K_{1}\right) \geqq m(R) \cdot\left(1-h^{-1} k\right)^{\nu}
$$

Define $\zeta_{1}=0$ on $\bar{R}_{1}$ and equal to $\zeta^{*}$ on $R^{*}$. Then

$$
\left|\zeta_{1}\left(x_{1}\right)-\zeta_{1}\left(x_{2}\right)\right| \leqq\left|x_{1}-x_{2}\right| \quad \text { if } x_{1} \in \bar{R}_{1}, \quad x_{2} \in R^{*} \text {. }
$$

Thus $\zeta_{1}$ satisfies a uniform Lipschitz condition with coefficient $M$ on $\bar{R}_{1} \cup R^{*}$. By a well known theorem, there exists an extension of $\zeta_{1}$ to $\bar{R}$ (the whole space in fact) which satisfies the same Lipschitz condition.

LEMma 2.2. Suppose the vectors $\zeta_{n} \rightarrow 0$ (in our sense) on $\bar{R}$ and suppose $f$ is quasi-convex in $p$. Then if $p_{0}$ is a constant vector we have

$$
m(R) f\left(p_{0}\right) \leqq \liminf _{n \rightarrow \infty} \int_{R} f\left[p_{0}+\pi_{n}(x)\right] d x
$$

Proof. For all sufficiently large $n$, we have $k_{n}<h$, and $k_{n} \rightarrow 0, k_{n}$ being the maximum of $\left|\zeta_{n}(x)\right|$ for $x \in R^{*}$. For each $n$ for which $k_{n}<h$, let $\eta_{n}$ be the 
function of the preceding lemma which coincides on $R^{*}$ with $\zeta_{n}$, and let $\omega_{n}$ $=\zeta_{n}-\eta_{n}$. Then if each $\zeta_{n}$ satisfies a uniform Lipschitz condition with coefficient $M \geqq 1$ on $\bar{R}$, then each $\eta_{n}$ and $\omega_{n}$ satisfies one with coefficient $M$ and $2 M$, respectively. Moreover, each derivative $\eta_{n x^{\alpha}}^{i}$ is uniformly bounded and $\eta_{n x \alpha}^{i} \rightarrow 0$ almost everywhere. Since $f$ is uniformly continuous on any bounded portion of $p$-space, we see that

$$
\lim _{n \rightarrow \infty} \int_{R}\left|f\left(p_{0 \alpha}^{i}+\eta_{n x \alpha}^{i}+\omega_{n x \alpha}^{i}\right)-f\left(p_{0 \alpha}^{i}+\omega_{n x \alpha}^{i}\right)\right| d x=0
$$

But the result then follows, since, for each $n$, we have

$$
\int_{R} f\left(p_{0 \alpha}^{i}+\omega_{n x \alpha}^{i}\right) \mathrm{d} \mathbf{x} \geqq m(R) f\left(p_{0}\right),
$$

because of the quasi-convexity of the function $f$.

THEовем 2.2. Suppose $f$ is continuous in $(x, z, p)$ for all $(x, z, p)$ and is quasi-convex in $p$ for each $(x, z)$. Suppose also that $z_{n} \rightarrow z_{0}$ on the bounded domain D. Then

$$
I\left(z_{0}, D\right) \leqq \liminf _{n \rightarrow \infty} I\left(z_{n}, D\right)
$$

Proof. Let $\epsilon$ be any positive number. For each positive integer $k$, let $D_{k}$ consist of all the hypercubes of edge $2^{-k}$ whose faces lie along hyperplanes $x^{\alpha}=2^{-k} i^{\alpha}$ (each $i^{\alpha}$ an integer) which lie in $D$. Since all the points $\left[x, z_{0}(x)\right.$, $\left.p_{0}(x)\right]$ and $\left[x, z_{n}(x), p_{n}(x)\right]$ for $x \in D$ lie in a bounded portion of $(x, z, p)$ space, we may choose $k_{1}$ so large that

$$
\int_{D-D_{k_{1}}}\left|f\left(x, z_{n}, p_{n}\right)\right| d x<\epsilon / 5, \int_{D-D_{k_{1}}}\left|f\left(x, z_{0}, p_{0}\right)\right| d x<\epsilon / 5
$$

for all $n$.

Let the hypercubes of $D_{k_{1}}$ be $R_{1}, \cdots, R_{N}$. For each $k \geqq k_{1}$, let $R_{k i}$, $i=1, \cdots, N \cdot 2^{\nu\left(k-k_{1}\right)}$, be all the hypercubes of side $2^{-k} \overline{\bar{d}}$ escribed above which lie in $D_{k_{1}}$. For each such $k$, define $x_{k}^{*}(x), z_{k}^{*}(x), p_{k}^{*}(x)$ on $D_{k_{1}}$ by

$$
\begin{aligned}
& x_{k}^{*}(x)=\left[m\left(R_{k i}\right)\right]^{-1} \int_{R_{k i}} x d x, z_{k}^{*}(x)=\left[m\left(R_{k i}\right)\right]^{-1} \int_{R_{k i}} z_{0}(x) d x, \\
& p_{k}^{*}(x)=\left[m\left(R_{k i}\right)\right]^{-1} \int_{R_{k i}} p_{0}(x) d x \\
& r_{k}(x)=\left\{\left|x_{k}^{*}(x)-x\right|^{2}+\left|z_{k}^{*}(x)-z_{0}(x)\right|^{2}+\left|p_{k}^{*}(x)-p_{0}(x)\right|^{2}\right\}^{1 / 2},
\end{aligned}
$$


where $\quad x \in R_{k i}$. Let $\zeta_{n}(x)=z_{n}(x)-z_{0}(x), \pi_{n}(x)=p_{n}(x)-p_{0}(x)$. Then, on $D_{k_{1}}$,

$$
\begin{aligned}
& f\left[x, z_{n}(x), p_{n}(x)\right]-f\left[x, z_{0}(x), p_{0}(x)\right] \\
= & \left\{f\left[x, z_{n}(x), p_{n}(x)\right]-f\left[x, z_{0}(x), p_{n}(x)\right]\right\} \\
+ & \left\{f\left[x, z_{0}(x), p_{0}(x)+\pi_{n}(x)\right]-f\left[x_{k}^{*}(x), z_{k}^{*}(x), p_{k}^{*}(x)+\pi_{n}(x)\right]\right\} \\
- & \left\{f\left[x, z_{0}(x), p_{0}(x)\right]-f\left[x_{k}^{*}(x), z_{k}^{*}(x), p_{k}^{*}(x)\right]\right\} \\
+ & \left\{f\left[x_{k}^{*}(x), z_{k}^{*}(x), p_{k}^{*}(x)+\pi_{n}(x)\right]-f\left[x_{k}^{*}(x), p_{k}^{*}(x), p_{k}^{*}(x)\right]\right\} .
\end{aligned}
$$

Now, all the arguments of $f$ occurring in (2.3) for $x \in D_{k_{1}}$ lie in a bounded closed cell in $(x, z, p)$-space over which $f$ is uniformly continuous. Let

$$
\epsilon(\rho)=\max \left|f\left(x^{\prime}, z^{\prime}, p^{\prime}\right)-f\left(x^{\prime \prime}, z^{\prime \prime}, p^{\prime \prime}\right)\right|, \quad \rho \geqq 0
$$

for all $\left(x^{\prime}, z^{\prime}, p^{\prime}\right)$ and $\left(x^{\prime \prime}, z^{\prime \prime}, p^{\prime \prime}\right)$ in this cell with

$$
\left|x^{\prime}-x^{\prime \prime}\right|^{2}+\left|z^{\prime}-z^{\prime \prime}\right|^{2}+\left|p^{\prime}-p^{\prime \prime}\right|^{2} \leqq \rho^{2} .
$$

then $\epsilon(\rho)$ is continuous for $\rho \geqq 0$ with $\epsilon(0)=0$. Then, for each $n$ and each $k \geqq k_{1}$, we have

$\left|f\left[x, z_{n}(x), p_{n}(x)\right]-f\left[x, z_{0}(x), p_{n}(x)\right]\right| \leqq \epsilon\left(\left|z_{n}(x)-z_{0}(x)\right|\right)$,

$\left|f\left[x, z_{0}(x), p_{0}(x)+\pi_{n}(x)\right]-f\left[x_{k}^{*}(x), z_{k}^{*}(x), p_{k}^{*}(x)+\pi_{n}(x)\right]\right| \leqq \epsilon\left[r_{k}(x)\right]$,

$\left|f\left[x, z_{0}(x), p_{0}(x)\right]-f\left[x_{k}^{*}(x), z_{k}^{*}(x), p_{k}^{*}(x)\right]\right| \leqq \epsilon\left[r_{k}(x)\right]$.

Now, the $r_{k}(x)$ are uniformly bounded on $D_{k_{1}}$ and tend to zero almost everywhere on $D_{k_{1}}$. Hence we may choose a $k \geqq k_{1}$ so large that

$\int_{D_{k_{1}}}\left|f\left[x, z_{0}(x), p_{0}(x)+\pi_{n}(x)\right]-f\left[x_{k}^{*}(x), z_{k}^{*}(x), p_{k}^{*}(x)+\pi_{n}(x)\right]\right| d x<\epsilon / 5$,

$\int_{D_{k_{1}}}\left|f\left[x, z_{0}(x), p_{0}(x)\right]-f\left[x_{k}^{*}(x), z_{k}^{*}(x), p_{k}^{*}(x)\right]\right| d x<\epsilon / 5$,

for all $n$. Since $z_{n}$ converges uniformly to $z_{0}$, there is an $n_{1}$ such that

$$
\int_{D_{k_{1}}}\left|f\left[x, z_{n}(x), p_{n}(x)\right]-f\left[x, z_{0}(x), p_{n}(x)\right]\right| d x<\epsilon / 5, n>n_{1} .
$$


Finally, since $x_{k}^{*}(x)$, and so on, are constant on each $R_{k i}$, and $f$ is quasi-convex, we conclude from the previous lemma that

$\liminf _{n \rightarrow \infty} \int_{D_{k_{1}}}\left\{f\left[x_{k}^{*}(x), z_{k}^{*}(x), p_{k}^{*}(x)+\pi_{n}(x)\right]-f\left[x_{k}^{*}(x), z_{k}^{*}(x), p_{k}^{*}(x)\right]\right\} d x \geqq 0$.

Using (2.3) - (2.5) and the above inequality, we see that

$$
\liminf _{n \rightarrow \infty} I\left(z_{n}, D\right) \geqq I\left(z_{0}, D\right)-\epsilon .
$$

Since $\epsilon$ is any positive number, the result follows.

3. Lower semicontinuity and weak convergence in $\Re_{s}(s \geqq 1)$. In this section, we discuss additional conditions which with the quasi-convexity of $f$ in $p$ are sufficient to guarantee the lower semicontinuity of $l(z, D)$ with respect to weak convergence in $\Re_{s}$ on $D$.

DEFINITION 3.1. Suppose $\zeta$ is of class $\Re_{s}$ on the bounded domain $D$ and suppose $R$ is a cell with $\bar{R} \subset D$. Then $\zeta$ is said to be strongly of class $\Re_{s}$ on $R^{*}$ if and only if $\bar{\zeta}$ is of class $\Re_{s}$ in $x_{\alpha}^{\prime}$ on each face $x^{\alpha}=$ const. of $R^{*}$ and there is a sequence $\zeta_{n}$ of class $C^{\prime}$ on $\bar{R}$ such that

$$
\bar{D}_{s}\left(\zeta_{n}-\zeta, R\right) \rightarrow 0, \bar{D}_{s}\left(\zeta_{n}-\bar{\zeta}, R^{*}\right) \rightarrow 0 \text {. }
$$

Lemma 3.1. Suppose $\zeta$ is of class $\Re_{s}(s \geqq 1)$ on the bounded domain D. For each $\alpha, 1 \leqq \alpha \leqq \nu$, let $\left(a^{\alpha}, b^{\alpha}\right)$ be the open interval projection of $D$ on the $x^{\alpha}$ axis. Then there exist sets $Z^{\alpha}$ of measure zero such that if $R: c^{\alpha} \leqq x^{\alpha} \leqq d^{\alpha}$ $(\alpha=1, \cdots, \nu)$ is any closed cell in $D$ with

$$
c^{\alpha} \in\left(a^{a}, b^{a}\right)-Z^{a}, \quad d^{a} \in\left(a^{a}, b^{\alpha}\right)-Z^{a} \quad(\alpha=1, \cdots, \nu),
$$

then $\zeta$ is strongly of class $\Re_{s}$ on $R^{*}$.

Proof. Let $R^{\prime}$ be any rational cell in $D$ (that is, $R=[C, D]$ with $C^{\alpha}, D^{\alpha}$ rational). In [1], Lemma 5.1, we have seen that if $\zeta$ is of class $\beta_{s}$ on $D$, then

$$
\lim _{h \rightarrow 0} \bar{D}_{s}\left(\zeta_{h}-\zeta, R\right)=0
$$

For each $\alpha$, define

$$
\phi_{h}^{\alpha}\left(x^{\alpha}, R^{\prime}\right)=\int_{C_{\alpha}^{\prime}}^{D_{\alpha}^{\prime}}\left\{\left|\zeta_{h}-\bar{\zeta}\right|^{s}+\left[\sum_{\substack{\beta=1 \\ \beta \neq \alpha}}^{\nu}\left|\zeta_{h x} \beta-\bar{\zeta}_{x}\right|^{2}\right] s / 2\right\} d x_{\alpha}^{\prime} .
$$


Since $\bar{\zeta}$ is obviously of class $\dddot{P}_{s}^{\prime}$ in $x_{\alpha}^{\prime}$ for almost all $x^{\alpha}$ on $\left[C^{\alpha}, D^{\alpha}\right], \phi_{h}^{\alpha}\left(x^{\alpha}, R^{\prime}\right)$ is defined for almost all $x^{\alpha}$ and

$$
\lim _{h \rightarrow 0} \int_{C^{\alpha}}^{D^{\alpha}}\left|\phi_{h}^{\alpha}\left(x^{\alpha}, R^{\prime}\right)\right| d x^{\alpha}=0 .
$$

By arranging the rational cells $R^{\prime}$ in some order and choosing successive subsequences, we may choose (on account of (3.1)) a final sequence $h_{n} \rightarrow 0$ such that $\phi_{h_{n}}^{\alpha}\left(x^{\alpha}, R^{\prime}\right) \rightarrow 0$ and $\bar{\zeta}$ is of class $\Re_{s}^{\prime}$ in $x_{\alpha}^{\prime}$ on $\left[C_{\alpha}^{\prime}, D_{\alpha}^{\prime}\right]$ for each $x^{\alpha}$ not in a set $Z^{\alpha}\left(R^{\prime}\right)$ of measure zero $(\alpha=1, \cdots, \nu)$. Now let

$$
Z^{a}=U Z^{a}\left(R^{\prime}\right)
$$

then

$$
m\left(Z^{\alpha}\right)=0 \quad(\alpha=1, \cdots, \nu) .
$$

Now suppose $R$ is one of the cells described in the lemma. Then it lies in some rational cell $R^{\prime}$ and we may take $\zeta_{n}=\zeta_{h_{n}}$.

Lemma 3.2. Suppose $R$ is a cell with edges $\left(2 h^{1}\right), \cdots,\left(2 h^{\nu}\right)$ and center $x_{0}$. Let

$$
h=\min _{1 \leqq \alpha \leqq \nu} h^{\alpha}, \quad K=h^{-1}\left(h^{\alpha} h^{\alpha}\right)^{1 / 2}
$$

Suppose also that $0<k<h$, that $\zeta^{*}$ is of class $\Re_{s}$ on an open domain containing $\bar{R}$ in its interior, and that $\zeta^{*}$ is strongly of class $\Re_{s}$ on $R^{*}$ with

$$
\int_{R^{*}}\left|\zeta^{*}\right|^{s} d S \leqq k^{s}, D_{s}\left(\zeta^{*}, R^{*}\right) \leqq M^{s} \quad(s \geqq 1) .
$$

Then there is a function $\zeta$ of class $\Re_{s}$ on $R$ which coincides with $\zeta^{*}$ on $R^{*}$, is zero except on a set of measure

$$
m(R) \cdot\left[1-\left(1-h^{-1} k\right)^{\nu}\right],
$$

and satisfies

$$
D_{s}(\zeta, R) \leqq \tau_{s} h^{-1} k\left(1+K^{s} M^{s}\right), \tau_{s}= \begin{cases}2^{s / 2} & (s \leqq 2), \\ 2^{s-1} & (s \geqq 2) .\end{cases}
$$

Proof. For each $x \in \bar{R}, x \neq x_{0}$, let $x^{*}(x)$ be the intersection of the ray $\overrightarrow{x_{0} x}$ with $R^{*}$, and for each $x \in \bar{R}$ define

$$
r(x)=\left\{\begin{array}{cc}
0 & \left(x=x_{0}\right), \\
\left|x^{*}(x)-x_{0}\right|^{-1} \cdot\left|x-x_{0}\right| & \left(x \neq x_{0}\right) .
\end{array}\right.
$$

Let $\Pi_{\alpha}^{ \pm}$be the pyramid in $\bar{R}$ with vertex $x_{0}$ and base the face $F_{\alpha}^{ \pm}$where 


$$
x^{\alpha}=x_{0}^{\alpha} \pm h^{\alpha} .
$$

On the pyramid $\Pi_{\nu}^{+}$, introduce coordinates $\xi^{1}, \cdots, \xi^{\nu-1}, r$ by

$$
x^{\nu}=x_{0}^{\nu}+r h^{\nu}, x^{\gamma}=x_{0}^{\gamma}+r \xi^{\gamma} \quad(0 \leqq r \leqq 1, \gamma=1, \cdots, \nu-1) .
$$

Then, if $r$ and $\xi^{\gamma}$ are considered as functions of $x$, we have

$$
r(x)=r, x^{*}(x)=\left[\xi^{1}(x)+x_{0}^{1}, \cdots, \xi^{\nu-1}(x)+x_{0}^{\nu-1}, h^{\nu}+x_{0}^{\nu}\right] .
$$

Similar coordinate systems may be set up on each of the other $\Pi_{a}^{ \pm}$.

Define

$$
\phi(r)= \begin{cases}0 & \left(0 \leqq r \leqq 1-k h^{-1}\right), \\ h k^{-1}\left(r-1+k h^{-1}\right) & \left(1-k h^{-1} \leqq r \leqq 1\right) .\end{cases}
$$

Choose a sequence $\zeta_{n}^{*}$ satisfying the conditions of Definition 3.1; and for each $n$, define

$$
\zeta_{n}(x)=\phi[r(x)] \cdot \zeta_{n}^{*}\left[x^{*}(x)\right]
$$

Then each $\zeta_{n}(x)$ is of class $D^{\prime}$ on $\bar{R}$.

We now compute the derivatives of $\zeta_{n}$ on each pyramid $\Pi_{a}^{ \pm}$taking $\Pi_{\nu}^{+}$as an example. Then

$$
\begin{array}{lr}
\zeta_{n x} \gamma=r^{-1} \phi(r) \zeta_{n \xi^{\gamma}}^{*} & (1 \leqq \gamma \leqq \nu-1), \\
\zeta_{n x^{\nu}}=\left(h^{\nu}\right)^{-1} \phi^{\prime}(r) \zeta_{n}^{*}-\left(h^{\nu}\right)^{-1} r \phi(r) \xi^{\gamma} \zeta_{n \xi^{\gamma}}^{*}(y \text { summed from } 1 \text { to } \nu-1) .
\end{array}
$$

Then, since $r^{-1} \phi(r) \leqq 1$ and $\phi^{\prime}(r)=k^{-1} h$ for $1-h k^{-1} \leqq r \leqq 1$,

$$
\begin{aligned}
\left|\pi_{n}(x)\right|^{2} & \leqq\left(\zeta_{n \xi}^{* i} \gamma \zeta_{n \xi}^{*_{i}} \gamma\right)+2 k^{-2}\left|\zeta_{n}^{*}\right|^{2}+2\left(h^{\nu}\right)^{-2}\left(\xi^{\gamma} \xi^{\gamma}\right)\left(\zeta_{n \xi}^{* i} \gamma \zeta_{n \xi^{\gamma}}^{* i}\right) \\
& \leqq 2\left[k^{-2}\left|\zeta_{n}^{*}\right|^{2}+K^{2}\left(\zeta_{n \xi^{\gamma}}^{* i} \zeta_{n \xi}^{* i}\right)\right] \quad \text { (n not summed). }
\end{aligned}
$$

Using the inequality

$$
\left(a^{2}+b^{2}\right)^{s / 2} \leqq \sigma_{s}\left(|a|^{s}+|b|^{s}\right), \quad \sigma_{s} \leqq \begin{cases}1 & (s \leqq 2) \\ 2^{(s-2) / 2} & (s \geqq 2)\end{cases}
$$


we obtain

$$
\begin{gathered}
D_{s}\left(\zeta_{n}, \Pi_{\nu}^{+}\right) \leqq \tau_{s} \int_{1-k h^{-1}}^{1} r^{\nu-1} d r \int_{F_{\nu}^{+}}\left[k^{-s}\left|\zeta_{n}^{*}\right|^{s}\right. \\
\left.+K^{s}\left(\zeta_{n \xi}^{* i} \gamma \zeta_{n \xi^{\gamma}}^{* i}\right)^{s / 2}\right] d s \\
\leqq \tau_{s} h^{-1} k\left[k^{-s} \int_{F_{\nu}^{+}}\left|\zeta_{n}^{*}\right|^{s} d s+K^{s} D_{s}\left(\zeta_{n}^{*}, F_{\nu}^{+}\right)\right] .
\end{gathered}
$$

Also

$$
\begin{aligned}
\int_{\Pi_{\nu}^{+}}\left|\zeta_{n}\right|^{s} d x & =\int_{1-k h^{-1}}^{1} r^{\nu-1} \phi^{s}(r) d r \int_{F_{\nu}^{+}}\left|\zeta_{n}^{*}\right|^{s} d S \\
& \leqq h^{-1} k \int_{F_{\nu}^{+}}\left|\zeta_{n}^{*}\right|^{s} d S .
\end{aligned}
$$

Adding these results for all the $\Pi_{\alpha}^{ \pm}$, we obtain the result for each $n$; and also $\bar{D}_{s}\left(\zeta_{n}, R\right)$ is uniformly bounded. Thus, we may extract a subsequence which tends weakly in $\Re_{s}$ to some function $\zeta$ of class $\Re_{s}$ on $R$. Since each $\zeta_{n}=\zeta_{n}^{*}$ on $R^{*}, \zeta_{n}^{*}$ tends strongly in $L_{s}$ to $\bar{\zeta}^{*}$ on $R^{*}$, we see from [2], Theorem 8.5 , that $\zeta=\bar{\zeta}^{*}$ on $R^{*}$. From the lower semicontinuity of $D_{s}$ (see [2], Theorem 8.2), the result follows.

LEMMA 3.3. Suppose $f$ is quasi-convex and of class $C^{\prime}$ for all $p$, and suppose for all $p$ that

$$
\sum_{i, \alpha}\left(f_{p_{\alpha}^{i}}\right)^{2} \leqq K^{2}\left(|p|^{s-1}+1\right)^{2}
$$

If $p_{0}$ is any constant vector, $D$ is any bounded domain, and $\zeta$ is of class $\Re_{s}$ on $D$ and vanishes on $D^{*}$, then $f\left[p_{0}+\pi(x)\right]$ is summable over $D$ and

$$
\int_{D} f\left[p_{0}+\pi(x)\right] d x \geqq m(D) \cdot f\left(p_{0}\right) .
$$

Proof. There exists a sequence of functions $\zeta_{n}$, each of class $C^{\prime}$ on $D$ and vanishing on and near $D^{*}$, such that $\bar{D}_{s}\left(\zeta_{n}-\zeta, D\right) \rightarrow 0$ (see [2], Definition 9.1). For each $n$ and almost all $x$ on $D$, we have

$$
\begin{aligned}
& \left|f\left[p_{0}+\pi_{n}(x)\right]-f\left[p_{0}+\pi(x)\right]\right|= \\
& \qquad\left[\pi_{n \alpha}^{i}(x)-\pi_{\alpha}^{i}(x)\right] \int_{0}^{1} f_{p_{\alpha}^{i}}\left[p_{0}+(1-t) \pi(x)+t \pi_{n}(x)\right] d t \mid
\end{aligned}
$$


$\leqq\left|\pi_{n}(x)-\pi(x)\right| \cdot K \cdot \int_{0}^{1}\left\{\left|(1-t) p_{0}+\pi(x)+t p_{0}+\pi_{n}(x)\right|-+1\right\} d t$

$\stackrel{(3.2)}{\lessgtr} K\left|\pi_{n}(x)-\pi(x)\right|\left\{h_{s}\left|p_{0}+\pi(x)\right|^{s-1}+h_{s}\left|p_{0}+\pi_{n}(x)\right|^{s-1}+1\right\}$,

where

$$
h_{s}= \begin{cases}s^{-1} & (1 \leqq s \leqq 2) \\ s^{-1} 2^{s-2} & (s \geqq 2) .\end{cases}
$$

Using the Hölder inequality, and so on, and the strong convergence in $\Re_{s}$, we see that

$$
\lim _{n \rightarrow \infty} \int_{D} f\left[p_{0}+\pi_{n}(x)\right] d x=\int_{D} f\left[p_{0}+\pi(x)\right] d x .
$$

Since $f$ is quasi-convex, the result follows.

Lemma 3.4. Suppose that $f$ satisfies the hypotheses of Lemma 3.3. Suppose also that each $\zeta_{n}$ is of class $\Re_{s}$ on a domain $D$ and is strongly of class $\Re_{s}$ on $R^{*}, \bar{R} \subset D$, with

$\lim _{n \rightarrow \infty} \int_{R^{*}}\left|\zeta_{n}\right|^{s} d S=0, D_{s}\left(\zeta_{n}, R^{*}\right) \leqq M^{s}, D_{s}\left(\zeta_{n}, R\right) \leqq M^{s} \quad(n=1,2 . \cdots)$

Then for each $p_{0}, f\left[p_{0}+\pi_{n}(x)\right]$ is summable for all sufficiently large $n$, and

$$
\liminf _{n \rightarrow \infty} \int_{R} f\left[p_{0}+\pi_{n}(x)\right] d x \geqq m(R) \cdot f\left(p_{0}\right), \pi_{n \alpha}^{i}(x)=\zeta_{n x^{\alpha}}^{i}(x) .
$$

Proof. For each $n$, let

$$
k_{n}=\left[\int_{R^{*}}\left|\zeta_{n}\right|^{s} d S\right]^{1 / s},
$$

and let $K$ and $h$ be the quantities of Lemma 3.2 for $R$. Since $k_{n} \rightarrow 0$, we have $k_{n}<h$ for all $n>$ some $n_{1}$. For each such $n$, let $\eta_{n}$ be the function of Lemma 3.2 which coincides on $R^{*}$ with $\zeta_{n}$, and let

$$
\chi_{n}=\zeta_{n}-\eta_{n}, \kappa_{n \alpha}^{i}=\eta_{n x^{a}}^{i}, \omega_{n \alpha}^{i}=\chi_{n x^{\alpha}}^{i} .
$$

Then, since $\chi_{n}=0$ on $R^{*}$, we have

$$
\int_{R} f\left[\pi_{0}+\omega_{n}(x)\right] d x \geqq m(R) f\left(p_{0}\right) .
$$


As in (3.2), we see that, for each $n$, and almost all $x$ on $D$,

$$
\begin{aligned}
& \left|f\left[p_{0}+\omega_{n}(x)+\kappa_{n}(x)\right]-f\left[p_{0}+\omega_{n}(x)\right]\right| \\
& \quad \leqq K \cdot\left|\kappa_{n}(x)\right| \cdot\left(h_{s}\left|p_{0}+\omega_{n}(x)+\kappa_{n}(x)\right|^{s-1}+h_{s}\left|p_{0}+\omega_{n}(x)\right|^{s-1}+1\right) \\
& \quad \leqq K \cdot\left|\kappa_{n}(x)\right| \cdot\left[\left(1+s h_{s}\right) h_{s}\left|p_{0}+\pi_{n}(x)\right|^{s-1}+s h_{s}^{2}\left|\kappa_{n}(x)\right|^{s-1}+1\right] .
\end{aligned}
$$

Using the Hölder inequality, and so on, we see that

$$
\lim _{n \rightarrow \infty} \int_{R}\left|f\left[p_{0}+\pi_{n}(x)\right]-f\left[p_{0}+\omega_{n}(x)\right]\right| d x=0,
$$

from which the result follows.

THEOREM 3.1. Suppose $f$ is of class $C^{\prime}$ in $(x, z, p)$ and quasi-convex in $p$. Suppose also that there are numbers $k$ and $K, K>0$, such that

(i) $f(x, z, p) \geqq k$,

(ii) $f_{p_{\alpha} i} f_{p_{\alpha}} \leqq K^{2}\left(|p|^{s-1}+1\right)^{2}$, for all $(x, z, p)$.

Suppose also that $z_{n} \rightarrow z_{0}$ weakly in $\Re_{s}$ on the bounded domain $D$ and that either

(a) each $z_{n}$ and $z_{0}$ are continuous on $D$ and $z_{n}$ converges uniformly to $z_{0}$ on each closed set interior to $D$, or

(b) the set functions $D_{s}\left(z_{n}, e\right)$ are uniformly absolutely continuous on each closed set interior to $D$.

Then

$$
I\left(z_{0}, D\right) \leqq \liminf _{n \rightarrow \infty} I\left(z_{n}, D\right)
$$

REMARK. If $s=1$, weak convergence in $\Re_{s}$ implies the hypothesis (b). Proof. We note first that hypothesis (ii) implies

$$
\begin{aligned}
& |f(x, z, p)-f(x, z, 0)|=\left|p_{\alpha}^{i} \int_{0}^{1} f_{p_{\alpha}^{i}}\left(x, z, t p_{\alpha}^{i}\right) d t\right| \\
& \quad \leq|p| \cdot \int_{0}^{1}\left[f_{p_{\alpha}^{i}} f_{p_{\alpha}^{i}}\left(x, z, t p_{\alpha}^{i}\right)\right]^{1 / 2} d t
\end{aligned}
$$




$$
\leqq|p| \cdot \int_{0}^{1} K\left(t^{s-1} p^{s-1}+1\right) d t \leqq K\left(s^{-1}|p|^{s}+|p|\right)
$$

Also, hypotheses (iii) and (iv) similarly imply

$$
|f(x, z, 0)-f(0,0,0)| \leqq K(|x|+|z|) .
$$

Thus, for all $(x, z, p)$, we have

$$
|f(x, z, p)| \leqq|f(0,0,0)|+K\left(|x|+|z|+s^{-1}|p|^{s}+|p|\right) .
$$

Therefore $I\left(z_{0}, D\right)$ and the $I\left(z_{n}, D\right)$ are uniformly bounded.

For each $\alpha(1 \leqq \alpha \leqq \nu)$, let $\left(a^{\alpha}, b^{\alpha}\right)$ be the open interval projection of $D$ on the $x^{\alpha}$ axis and let $Z_{0}^{\alpha}$ and $Z_{n}^{a}$ be the sets of Lemma 3.1 for $z_{0}$ and $z_{n}$. Also for each $\alpha, n, k$, let $E_{n, k}^{\alpha}$ be the set of $x^{\alpha}$ in $\left(a^{\alpha}, b^{\alpha}\right)-Z_{n}^{\alpha}$, where

$$
\bar{D}_{s}\left(\bar{z}_{n}, D_{x^{a}}\right) \leqq k,
$$

$D_{x^{\alpha}}$ being the set of $x_{\alpha}^{\prime}$ such that $\left(x_{\alpha}^{\prime}, x^{\alpha}\right) \in D$. Suppose that $\bar{D}_{s}\left(z_{n}, D\right) \leqq M$, some uniform bound existing because of the weak convergence. Let

$$
Z_{n, k}^{a}=\left(a^{\alpha}, b^{\alpha}\right)-E_{n, k}^{a}
$$

Then

$$
m\left(Z_{n, k}^{a}\right) \leqq M k^{-1}, m\left(E_{n, k}^{\alpha}\right) \geqq\left(b^{a}-a^{\alpha}\right)-M k^{-1} .
$$

For each $\alpha$, let

$$
E^{a}=\bigcup_{k=1}^{\infty} \bigcap_{N=1}^{\infty} \bigcup_{n=N}^{\infty} E_{n, k}^{\alpha}, \tilde{Z}_{0}^{\alpha}=\left(a^{\alpha}, b^{\alpha}\right)-E^{\alpha} \cup Z_{0}^{a} \cup \bigcup_{n=1}^{\infty} Z_{n}^{a} .
$$

Then $m\left(Z_{0}^{a}\right)=0$. For each $\alpha$, each natural number $n$, and each integer $i$, define $Z_{n, i}^{a}$ as the set of all $x^{a}$ such that $x^{a}-i \cdot 2^{-n} \in \tilde{Z}_{0}^{a}$, and define

$$
Z^{a}=\bigcup_{n, i} Z_{n, i}^{a}
$$

Then $m\left(Z^{a}\right)=0$.

Now, choose a point $x_{0}$ such that $x_{0}^{a}$ is not in $Z^{\alpha}(\alpha=1, \cdots, \nu)$. For each natural number $k$, let $\oint_{k}$ be the totality of hypercubes of side $2^{-k}$ bounded by hyperplanes of the form $x^{a}=x_{0}^{a}+i \cdot 2^{-k}$. None of the numbers $x_{0}^{a}+i \cdot 2^{-k}$ is in $Z^{\alpha}$ and, moreover, $\bar{z}_{0}$ and each $\bar{z}_{n}$ is strongly of class $\Re_{s}$ on $R^{*}$ with 
$\bar{D}_{s}\left(\bar{z}_{n}, R\right)$ uniformly bounded for infinitely many values of $n, R$ being any hypercube of any $G_{k}$. Since the totality of these hypercubes is countable, we may choose a subsequence, still called $z_{n}$, such that $I\left(z_{n}, D\right)$ tends to the former lim inf, $\bar{z}_{n} \rightarrow \bar{z}_{0}$ almost everywhere on $D$, and $\bar{D}_{s}\left(z_{n}, R^{*}\right)$ is uniformly bounded for $R$ of any $C_{k}$ in $D$. Since $z_{n} \rightarrow z$ in $\mathbb{P}_{s}$, we also have

$$
\lim _{n \rightarrow \infty} \int_{R^{*}}\left|z_{n}-z_{0}\right|^{s} d S=0
$$

for each such $R$.

Now, we first consider the alternative (a). Let $\epsilon$ be any positive number. For each $k$, let $D_{k}$ be the union of all the cells of $C_{k}$ which are interior to $D$. Since $f$ is bounded below and $l\left(z_{0}, D\right)$ is finite, we first choose $k_{1}$ so large that

$$
I\left(z_{n}, D-D_{k_{1}}\right)>-\epsilon / 5 \quad(n=1,2, \cdots) .
$$

$$
I\left(z_{0}, D_{k_{1}}\right)>I\left(z_{0}, D\right)-\epsilon / 5 .
$$

For this $k_{1}$, let $R_{1}, \ldots, R_{q}$ be the cells of $D_{k_{1}}$ and for each $k \geqq k_{1}$, let

$$
R_{k i} \quad\left(i=1, \cdots, q \cdot 2^{\nu\left(k-k_{1}\right)}\right)
$$

be the cells of $\mathscr{f}_{k}$ in $D_{k_{1}}$ For each $k$, define $x_{k}^{*}(x), z_{k}^{*}(x)$, and $p_{k}^{*}(x)$ on $D_{k_{1}}$ by (2.9). Then, from (ii), (iii), and (iv), it follows that

$$
\begin{aligned}
& \left|f\left[x, z_{0}(x), p_{0}(x)\right]-f\left[x_{k}^{*}(x), z_{k}^{*}(x), p_{k}^{*}(x)\right]\right| \\
& \quad \leqq K\left(\left|p_{0}(x)\right|^{s}+1\right) \cdot\left(\left|x-x_{k}^{*}(x)\right|+\left|z_{0}(x)-z_{k}^{*}(x)\right|\right) \\
& \quad+K\left(h_{s}\left|p_{0}(x)\right|^{s-1}+h_{s}\left|p_{k}^{*}(x)\right|^{s-1}+1\right) \cdot\left|p_{0}(x)-p_{k}^{*}(x)\right|,
\end{aligned}
$$

where

$$
h_{s}= \begin{cases}s^{-1} & (1 \leqq s \leqq 2), \\ s^{-1} \cdot 2^{s-2} & (s \geqq 2) ;\end{cases}
$$

the method of proof is similar to that of (3.3). If we let

$$
\zeta_{n}=z_{n}-z_{0}, \pi_{n}=p_{n}-p_{0},
$$

we see similarly that 


$$
\left|f\left[x, z_{0}(x), p_{0}(x)+\pi_{n}(x)\right]-f\left[x_{k}^{*}(x), z_{k}^{*}(x), p_{k}^{*}(x)+\pi_{n}(x)\right]\right|
$$

.8) $\leqq K\left(\left|p_{n}(x)\right|^{s}+1\right)\left(\left|x-x_{k}^{*}(x)\right|+\left|z_{0}(x)-z_{k}^{*}(x)\right|\right)$

$$
+K\left(h_{s}\left|p_{n}(x)\right|^{s-1}+h_{s}\left|p_{k}^{*}(x)+\pi_{n}(x)\right|^{s-1}+1\right) \cdot\left|p_{0}(x)-p_{k}^{*}(x)\right| ;
$$

(3.9) $\left|f\left[x, z_{n}(x), p_{n}(x)\right]-f\left[x, z_{0}(x), p_{n}(x)\right]\right|$

$$
\leqq K\left(\left|p_{n}(x)\right|^{s}+1\right) \cdot\left|z_{n}(x)-z_{0}(x)\right|
$$

Now, by the Hölder inequality on each $R_{k i}$, we see that

$$
\int_{D_{k_{1}}}\left|p_{k}^{*}(x)\right|^{s} d x \leqq \int_{D_{k_{1}}}\left|p_{0}(x)\right|^{s} d x
$$

By applying the Minkowski inequality, we see that the integrals

$$
\int_{D_{k_{1}}}\left|\pi_{n}(x)\right|^{s} d x, \int_{D_{k_{1}}}\left|p_{k}^{*}(x)+\pi_{n}(x)\right|^{s} d x
$$

are uniformly bounded. Finally,

$$
\lim _{k \rightarrow \infty} \int_{D_{k_{1}}}\left|p_{0}(x)-p_{k}^{*}(x)\right|^{s} d x=0
$$

Hence, using (3.7)-(3.12), we may choose a $k$ so large that

$$
\begin{array}{r}
\int_{D_{k_{1}}}\left|f\left[x, z_{0}(x), p_{0}(x)\right]-f\left[x_{k}^{*}(x), z_{k}^{*}(x), p_{k}^{*}(x)\right]\right| d x<\epsilon / 5, \\
\int_{D_{k_{1}}}\left|f\left[x, z_{0}(x), p_{n}(x)\right]-f\left[x_{k}^{*}(x), z_{k}^{*}(x) p_{k}^{*}(x)+\pi_{n}(x)\right]\right| d x<\epsilon / 5 \\
(n=1,2, \cdots),
\end{array}
$$

and then choose $n_{1}$ so large that

$$
\text { (3.15) } \int_{D_{k_{1}}}\left|f\left[x, z_{n}(x), p_{n}(x)\right]-f\left[x, z_{0}(x), p_{n}(x)\right]\right| d x<\epsilon / 5, \quad n>n_{1} \text {. }
$$

Since $x_{k}^{*}(x), z_{k}^{*}(x), p_{k}^{*}(x)$ are constant on each $R_{k i}$, it follows from Lemma 3.4 that

$$
\liminf _{n \rightarrow \infty} \int_{D_{k_{1}}} f\left[x_{k}^{*}(x), z_{k}^{*}(x), p_{k}^{*}(x)+\pi_{n}(x)\right] d x
$$




$$
\geqq \int_{D_{k_{1}}} f\left[x_{k}^{*}(x), z_{k}^{*}(x), p_{k}^{*}(x)\right] d x
$$

Using (3.6) and (3.13)-(3.16), we see that

$$
\left.\liminf _{n \rightarrow \infty} I\left(z_{n}, D\right) \geqq I_{0}, D\right)-\epsilon .
$$

The result follows in this case.

We now consider the alternative (b). For each nat ural number $q$, we define

$$
\begin{gathered}
f_{q}(x, z, p)=\left[1-a_{q}(x, z)\right] f(x, z, p)+k \cdot a_{q}(x, z), \\
a_{q}(x, z)= \begin{cases}0 & (0 \leqq R \leqq q), \\
3(R-q)^{2}-2(R-q)^{3} & (q \leqq R \leqq q+1), \\
1 & (R \geqq q+1), \quad R=\left(|x|^{2}+|z|^{2}\right)^{1 / 2} .\end{cases}
\end{gathered}
$$

Remembering (3.3)-(3.5), we see that each $f_{q}$ satisfies hypotheses (i) - (iv) with the same $k$ and some $K_{q}$. Moreover $f_{q}$ is independent of $(x, z)$ for $R \geqq q+1$, and also

$$
f_{q}(x, z, p) \leqq f_{q+1}(x, z, p), \lim _{q \rightarrow \infty} f_{q}(x, z, p)=f(x, z, p)
$$

Thus it is sufficient to prove the lower semicontinuity for each $q$.

For a fixed $q$, we note that we may replace $\left|z_{0}(x)-z_{k}^{*}(x)\right|$ by $\phi_{k}(x)$ in (3.7) and (3.8) and $\left|z_{n}(x)-z_{0}(x)\right|$ by $\psi_{n}(x)$ in (3.9), where

$$
\begin{aligned}
& \phi_{k}(x)=\min \left(\left|z_{0}(x)-z_{k}^{*}(x)\right|, 2 q+2\right), \\
& \psi_{n}(x)=\min \left(\left|z_{n}(x)-z_{0}(x)\right|, 2 q+2\right) .
\end{aligned}
$$

From the uniform boundedness of the $\phi_{k}$ and $\psi_{n}$ ( $q$ fixed), the uniform absolute continuity of the set function $D_{s}\left(z_{n}, e\right)$, and the facts that

$$
\lim _{k \rightarrow \infty} \phi_{k}(x)=0, \lim _{n \rightarrow \infty} \psi_{n}(x)=0
$$

almost everywhere, it follows that the argument can be carried through as before for each fixed $q$.

THEOREM 3.2. Suppose $s>\nu$ and suppose $f$ satisfies the hypotheses of Theorem 3.1 with (i) replaced by 


$$
f(x, z, p) \geqq m|p|^{s}+k \quad(m>0) .
$$

If $z^{*}$ is any function of class $\Re_{s}$ on the bounded domain $D$, then there is a function $z_{0}$ of class $\mathfrak{S}_{s}$ which coincides with $z^{*}$ on $D^{*}$ and minimizes $I(z, D)$ among all such functions.

Proof. Let $z_{n}$ be a minimizing sequence. It follows from ( $\left.\mathrm{i}^{\circ}\right)$ that $D_{s}\left(z_{n}, D\right)$ is uniformly bounded. From [2], Theorem 9.4, it follows that $\bar{D}_{s}\left(z_{n}, D\right)$ is uniformly bounded. But then a subsequence, still called $\left\{z_{n}\right\}$, converges weakly in $\Re_{s}$ to some function $z_{0}$ of class $\Re_{s}$ which coincides with $z^{*}$ on $D^{*}$ by [2], Theorem 9.2. But, from [3], Chapter II, Theorem 2.1, it follows that the equivalent functions $\bar{z}_{n}$ and $\bar{z}_{0}$ are equicontinuous on closed sets interior to $D$. Hence $z_{n}$ converges uniformly to $\bar{z}_{0}$ on each closed set interior to $D$. Hence, from the precerling theorem, $z_{0}$ is a desired solution.

More general theorems involving variable boundary values, similar to those in [3], Chapter III, §5, with $s>\nu$, can be proved.

4. Necessary conditions for quasi-convexity. In the two preceding sections, we have established the connection between quasi-convexity and lower semicontinuity. In this section, we shall establish some necessary conditions for quasi-convexity. In the next section, we establish some sufficient conditions which are also necessary when $f$ has certain interesting special forms. Unfortunately, the writer is unable to establish conditions which are both necessary and sufficient in the general case.

Lemma 4.1. Suppose $f$ is continuous, $Q$ is the cell

$$
\left|x^{\alpha}\right| \leqq 1 \quad(\alpha=1, \cdots, \nu), \delta>0
$$

and suppose

$$
\int_{Q} f[p+\pi(x)] d x \geqq f(p) \cdot m(Q)
$$

for every function $\zeta$ which satisfies a Lipschitz condition with coefficient $<\delta$ on $\bar{Q}$ and vanishes on $Q^{*}$. Then (4.1) also holds with $Q$ replaced by any bounded domain $D$.

Proof. Suppose $\zeta$ satisfies the conditions on the bounded domain $\bar{D}$. Let $\bar{R}$ be a hypercube of side $h$ which contains $\bar{D}$, and extend $\zeta$ to $\bar{R}$ :

$$
x_{0}^{\alpha} \leqq x^{\alpha} \leqq x_{0}^{\alpha}+h
$$


by defining $\zeta=0$ on $\bar{R}-\bar{D}$. Then $\zeta$ satisfies the conditions on $\bar{R}$, and

$$
\zeta^{*}(x)=h^{-1} \zeta\left(x_{0}+h x\right)
$$

satisfies the conditions on $\bar{Q}$, and

$$
\zeta_{x^{a}}^{* i}(x)=\zeta_{x^{a}}^{i}\left(x_{0}+h x\right)
$$

DEFINITION 4.1. The function $f$ is said to be weakly quasi-convex if with each $p$ is associated a $\delta_{p}>0$ such that (4.1) holds for all $D$ and all $\zeta$ satisfying a Lipschitz condition with coefficient $<\delta_{p}$ and vanishing on $D^{*}$.

In other words, $f$ is weakly quasi-convex if and only if each linear function furnishes a weak relative minimum among all Lipschitzian functions coinciding with it on the boundary, whereas $f$ is quasi-convex if and only if any linear function furnishes the absolute minimum among all such functions. Thus we have the following result.

THEOREM 4.1. If $f$ is continuous and quasi-convex, it is weakly quasiconvex.

We shall see that if $f$ is weakly quasi-convex and continuous, then $f$ satisfies a uniform Lipschitz condition on any bounded set in p-space and satisfies a generalized Weierstrass condition (see Theorem 4.3) which reduces to the ordinary Weierstrass condition if $f$ is of class $C^{\prime}$ (see (4.7)) and is equivalent to the Legendre-Hadamard condition (see (4.8)) if $f$ is of class $C^{\prime \prime}$.

Lemma 4.2. Suppose $\phi$ is continuous, and suppose corresponding to any point $\lambda_{0}$ in $E_{\nu}$ there is a $\delta>0$ such that for any unit vector $\mu$ we have

$$
k \phi\left(\lambda_{0}-h_{\mu}\right)+h \phi\left(\lambda_{0}+k \mu\right) \geqq(h+k) \phi\left(\lambda_{0}\right) \quad(0<h<\delta, 0<k<\delta) .
$$

Then $\phi$ is convex in $\lambda$.

Proof. Let $\lambda_{0}$ be any point, and $\mu$ any point with $|\mu|=1$. We shall show that

$$
\psi(t)=\phi\left(\lambda_{0}+\mu t\right)
$$

is convex in $t$. From the hypothesis, it follows that for each $t_{0}$, there is a $\delta\left(t_{0}\right)>0$ such that

$$
k \psi\left(t_{0}-h\right)+h \psi\left(t_{0}+k\right) \geqq(h+k) \psi\left(t_{0}\right) \quad(0 \leqq h \leqq \delta, \quad 0 \leqq k \leqq \delta) .
$$


Now, suppose $t_{1}<t_{2}$. Let

$$
\chi(t)=\psi(t)-\psi\left(t_{1}\right)-\frac{t-t_{1}}{t_{2}-t_{1}}\left[\psi\left(t_{2}\right)-\psi\left(t_{1}\right)\right] .
$$

Then $\chi(t)$ satisfies (4.2) and $\chi\left(t_{1}\right)=\chi\left(t_{2}\right)=0$. Suppose $M=\max \chi(t)$ $\left(t_{1} \leqq t \leqq t_{2}\right)$, and suppose $M>0$. Let $t_{0}$ be the smallest value of $t$ such that $\chi(t)=M$, and let the number $\delta\left(t_{0}\right)$ be chosen as above. Clearly $t_{1}<t_{0}<t_{2}$. Choose $t_{3}$ and $t_{4}$ with

$$
\left|t_{3}-t_{0}\right|<\delta,\left|t_{4}-t_{0}\right|<\delta \quad\left(t_{1} \leqq t_{3}<t_{0}<t_{4} \leqq t_{2}\right)
$$

Then $\chi\left(t_{3}\right)<M, \quad \chi\left(t_{4}\right) \leqq M$, so that

$$
\left(t_{4}-t_{0}\right) \times\left[t_{0}-\left(t_{0}-t_{3}\right)\right]+\left(t_{0}-t_{3}\right) \times\left[t_{0}+\left(t_{4}-t_{0}\right)\right]<\left(t_{4}-t_{3}\right) \times\left(t_{0}\right),
$$

which contradicts the hypothesis. Thus $\chi(t) \leqq 0$, so that

$$
\psi(t) \leqq \psi\left(t_{1}\right)+\frac{t-t_{1}}{t_{2}-t_{1}} \quad\left[\psi\left(t_{2}\right)-\psi\left(t_{1}\right)\right] .
$$

Since $t_{1}$ and $t_{2}$ were arbitrary with $t_{1}<t_{2}$, the function $\psi$ is convex in $t$. Thus $\phi$ is convex in $\lambda$.

THEOREM 4.2. If $f$ is weakly quasi-convex, then $f\left(p_{\alpha}^{i}+\lambda_{\alpha} \xi^{i}\right)$ is convex in $\lambda$ for each fixed $p$ and $\xi$.

Proof. Let $p_{\alpha}^{i}, \xi^{i}$ and $\lambda_{0 \alpha}$ be fixed and let $\mu_{1}$ be any unit vector, and suppose $h>0, k>0$. Choose $\delta\left(p_{\alpha}^{i}, \xi^{i}, \lambda_{0 \alpha}\right)>0$ but so small that, for any bounded domain $G$,

$$
\int_{G} f\left[p_{\alpha}^{i}+\lambda_{0 \alpha} \xi^{i}+\zeta_{x^{\alpha}}^{i}(x)\right] d x \geqq m(G) f\left(p_{\alpha}^{i}+\lambda_{0 \alpha} \xi^{i}\right)
$$

for all $\zeta$ satisfying a Lipschitz condition of constant $<\delta$ on $G$ and vanishing on $G^{*}$. Let $\left(\mu_{1}, \cdots, \mu_{\nu}\right)$ be a normal orthogonal set of unit vectors. If $\xi=0$, the result is obvious. If $\xi \neq 0$, choose $h$ and $k$ with $0<h|\xi|<\delta, 0<k|\xi|<\delta$, and let $\rho$ be any number $>|\xi| / \delta$. Let $H=(1 / \rho) k, K=(1 / \rho) h$, and let $R$ be the rectangular parallelepiped

$$
-\rho H \leqq y^{1} \leqq \rho K,\left|y^{\beta}\right| \leqq \rho \quad(\beta=2, \cdots, \nu)
$$


where

$$
y^{\beta}=x, \mu_{\beta}
$$

Let $F_{1}^{-}$be the face $y^{1}=-\rho H, F_{1}^{+}$be the face $y^{1}=\rho K, F_{\beta}^{-}$be the face $y^{\beta}=-\rho, F_{\beta}^{+}$be the face $y^{\beta}=\rho$, and let $\Pi_{\beta}^{-}$and $\Pi_{\beta}^{+}$be the pyramids with vertex at the origin and base $F_{\beta}^{-}$and $F_{\beta}^{+}$, respectively. Let $\zeta$ be defined on $\bar{R}$ to be continuous on $\bar{R}$, zero on $R^{*}$, linear on each $\Pi_{\beta}$ and $\Pi_{\beta}^{+}$, with $\zeta(0)=\xi$. Then

$$
\zeta_{x^{\alpha}}^{i}=\left\{\begin{array}{lr}
(\rho H)^{-1} \mu_{1 \alpha} \xi^{i}=k_{\mu_{1 \alpha}} \xi^{i} & , \text { on } \Pi_{1}^{-} \\
-(\rho K)^{-1} \mu_{1 \alpha} \xi^{i}=-h_{\mu_{1 \alpha}} \xi^{i}, & \text { on } \Pi_{1}^{+} \\
\rho^{-1} \mu_{\beta \alpha} \xi^{i} & , \text { on } \Pi_{\beta}^{-} \\
-\rho^{-1} \mu_{\beta \alpha} \xi^{i} & , \text { on } \Pi_{\beta}^{+}
\end{array}\right.
$$

Also

$$
m\left(\Pi_{1}^{-}\right)=\nu^{-1} 2^{\nu-1} \rho^{\nu} H, m\left(\Pi_{1}^{+}\right)=\nu^{-1} 2^{\nu-1} \rho^{\nu} K, m(R)=2^{\nu-1} \rho^{\nu}(H+K)
$$

$$
m\left(\mathrm{I}_{\beta}^{-}\right)=m\left(\Pi_{\beta}^{+}\right)=\nu^{-1} 2^{\nu-2} \rho^{\nu}(H+K) \quad(\beta=2, \cdots, \nu) .
$$

Then, by applying (4.3), (4.4), and (4.5), we obtain

$$
\begin{aligned}
& \frac{1}{2 \nu}\left\{\frac{2 k}{h+k} f\left[p_{\alpha}^{i}+\left(\lambda_{0 a}-h \mu_{1 \alpha}\right) \xi^{i}\right]+\frac{2 h}{h+k} f\left[p_{\alpha}^{i}+\left(\lambda_{0 \alpha}+k \mu_{1 \alpha}\right) \xi^{i}\right]\right. \\
& \left.+\sum_{\beta=2}^{\nu}\left(f\left[p_{\alpha}^{i}+\left(\lambda_{0 \alpha}-\rho^{-1} \mu_{\beta a}\right) \xi^{i}\right]+f\left[p_{\alpha}^{i}+\left(\lambda_{0 \alpha}+\rho^{-1} \mu_{\beta a}\right)\right]\right)\right\} \\
& \geqq f\left(p_{\alpha}^{i}+\lambda_{0 \alpha} \xi^{i}\right) .
\end{aligned}
$$

Letting $\rho \rightarrow \infty$, we obtain

$$
k f\left[p_{a}^{i}+\left(\lambda_{0 a}-h \mu_{1 a}\right) \xi^{i}\right]+h f\left[p_{a}^{i}+\left(\lambda_{0 a}+k \mu_{1 \alpha}\right) \xi^{i}\right] \geqq(h+k) f\left(p_{\alpha}^{i}+\lambda_{0 \alpha} \xi^{i}\right)
$$

From the preceding lemma, it follows that $f\left(p_{\alpha}^{i}+\lambda_{\alpha} \xi^{i}\right)$ is convex in $\lambda$ for each $\xi$ and $p$. 
THEOREM 4.3. Suppose $f$ is continuous and convex in $\lambda$ for $p$ and $\xi$.

Then $f$ satisfies a uniform Lipschitz condition on each bounded closed set, and for each fixed $p$ there exists a set of constants $A_{i}^{\alpha}$ such that

$$
f\left(p_{\alpha}^{i}+\lambda_{\alpha} \xi^{i}\right) \geqq f(p)+A_{i}^{\alpha} \lambda_{\alpha} \xi^{i}
$$

for all $\lambda$ and $\xi$. If $f$ is of class $C^{\prime}$, (4.6) holds if and only if $A_{i}^{\alpha}=f_{p_{\alpha}^{i}}$, that is,

$$
f\left(p_{\alpha}^{i}+\lambda_{\alpha} \xi^{i}\right) \geqq f(p)+f_{p_{\alpha}^{i}}(p) \lambda_{\alpha} \xi^{i} .
$$

If $f$ is of class $C^{\prime \prime},(4.7)$ holds for all $p, \lambda, \xi$ if and only if

$$
f_{p_{a}^{i} p_{\beta}^{j}}(p) \lambda_{a} \lambda_{\beta} \xi^{i} \xi^{j} \geqq 0
$$

for all $\lambda, \xi, p$.

Proof. Suppose, first, that $f$ is of class $C^{\prime}$. Let $p$ and $\xi$ be fixed. Then (4.7) follows from the convexity in $\lambda$. Moreover, since each unit vector $e_{\alpha}^{i}$ in the $p$-space is of the form $\lambda_{\alpha} \xi^{i}$, we see from the convexity in $\lambda$ that

$$
f(p)-f\left(p-e_{\alpha}^{i}\right) \leqq f_{p_{\alpha}^{i}}(p) \leqq f\left(p+e_{\alpha}^{i}\right)-f(p)
$$

for all $p$. Thus the derivatives of $f$ are uniformly bounded by these differences in the values of $f$ on any bounded part of space. Moreover, in this case, if constants $A_{i}^{\alpha}$ satisfy (4.6), we must have

$$
A_{i}^{a}=f_{p_{a}^{i}}(p) .
$$

Now, if $f$ is of class $C^{\prime \prime}$, equation (4.8) with $p$ replaced by $p_{a}^{i}+\lambda_{a} \xi^{i}$ is equivalent to the condition that $f$ is convex in $\lambda$ for each fixed $p$ and $\xi$.

Finally, if $f$ is continuous and has this stated convexity property, it is clear that the $h$-average function also does, and $f_{h}$ is of class $C^{\prime}$. By letting $h \rightarrow 0$, we see that $f$ satisfies a uniform Lipschitz condition on any bounded closed set. Now, choose $h_{n}=n^{-1}$ and choose $p$ fixed. From (4.9) and the uniform convergence of $f_{h}$ to $f$ on any bounded part of space, we conclude that the derivatives $f_{h_{n}} p_{a}^{i}(p)$ are uniformly bounded. We may therefore choose a subsequence, still called $h_{n}$, such that

$$
\lim _{n \rightarrow \infty} f_{h_{n} p_{\alpha}^{i}}(p)=A_{i}^{\alpha}
$$


Since (4.7) holds for all $\lambda$ and $\xi$ for each $n$, (4.6) holds in the limit.

5. Sufficient conditions for quasi-convexity. In this section we prove one general sufficient condition and then give conditions which are necessary and sufficient when $f$ has certain interesting special forms.

Lemma 5.1. Suppose $\zeta$ satisfies a uniform Lipschitz condition on the closure $\bar{D}$ of the bounded domain $D$ and suppose $\zeta=0$ on $D^{*}$. If

$$
1 \leqq \mu \leqq \nu, 1 \leqq i_{1}, \cdots, i_{\mu} \leqq N, 1 \leqq \alpha_{1}<\alpha_{2}<\cdots<\alpha_{\mu} \leqq \nu,
$$

then

$$
\int_{D} \frac{\partial\left(\zeta^{i_{1}}, \cdots, \zeta^{i_{\mu}}\right)}{\partial\left(x^{a_{1}}, \cdots, x^{\alpha_{\mu}}\right)} d x=0
$$

Proof. Choose a large cell $R$ containing $\bar{D}$ in its interior, and extend $\zeta$ by defining it to be zero outside $\bar{D}$. Then the second $h$-average function $\zeta_{h h}$ is of class $C^{\prime \prime}$ on $\bar{R}$ and vanishes on and near $R^{*}$. Since any integral of the above type formed for $\zeta_{h h}$ tends to that for $\zeta$ as $h \rightarrow 0$, we need prove the theorem only for functions $\zeta$ of class $C^{\prime \prime}$ on cells $\bar{R}$.

As an example, take $i_{\beta}=\alpha_{\beta}=\beta, \beta=1, \cdots, \mu, D=R$. Then

$$
\begin{aligned}
\int_{R} \frac{\partial\left(\zeta^{1}, \cdots, \zeta^{\mu}\right)}{\partial\left(x^{1}, \ldots, x^{\mu}\right)} d x & =\int_{R} \sum_{\alpha=1}^{\mu}(-1)^{\mu+\alpha} \zeta_{x^{\alpha}}^{\mu} Q d x \\
& =\int_{R^{*}} \zeta^{\mu} \sum_{\alpha=1}^{\mu}(-1)^{\mu+\alpha} Q d x_{\alpha}^{\prime} \\
& -\int_{R}(-1)^{\mu} \zeta^{\mu} \sum_{\alpha=1}^{\mu}(-1)^{\alpha} \frac{\partial}{\partial x^{\alpha}} Q d x,
\end{aligned}
$$

where

$$
Q=\frac{\partial\left(\zeta^{1}, \cdots, \zeta^{\alpha-1}, \zeta^{\alpha}, \cdots, \zeta^{\mu-1}\right)}{\partial\left(x^{1}, \cdots, x^{\alpha-1}, x^{\alpha+1}, \cdots, x^{\mu}\right)},
$$

the last equality holding by Green's theorem. But the boundary integral vanishes since $\zeta=0$ on $R^{*}$, and the integrand in the second integral vanishes on $R$ (see [3], Chapter II, Lemma 1.1, for instance). 
THEOREM 5.1. A sufficient condition that $f$ be quasi-convex is that for each $p$ there exist alternating forms

$$
A_{i}^{\alpha} \pi_{a}^{i}, A_{i j}^{\alpha \beta} \pi_{a}^{i} \pi_{\beta}^{j}, \cdots, A_{i_{1}}^{\alpha_{1}}, \cdots, \alpha_{\nu} \pi_{\alpha_{1}} \pi_{\nu} \ldots \pi_{\alpha_{\nu}}^{i_{\nu}}
$$

such that for all $\pi$ we have

$$
f(p+\pi) \geqq f(p)+A_{i}^{\alpha} \pi_{\alpha}^{i}+\cdots+A_{i_{1}}^{a_{1}}, \cdots, a_{\nu} \pi_{\alpha_{1}}^{i_{1}} \ldots \pi_{\alpha_{\nu}}^{i_{\nu}} .
$$

Proof. This is an immediate consequence of the preceding lemma.

THEOREM 5.2. If the $a_{i j}^{a \beta}$ are constants and

$$
f(p)=a_{i j}^{\alpha \beta} p_{\alpha}^{i} p_{\beta}^{j},
$$

a necessary and sufficient condition that $f$ be quasi-convex is that

$$
a_{i j}^{\alpha \beta} \lambda_{\alpha} \lambda_{\beta} \xi^{i} \xi^{j} \geqq 0
$$

for all $\lambda$ and $\xi$.

Proof. If $\zeta=0$ on $D^{*}$, we see from Lemma 5.1 that

$$
\int_{D} f[p+\pi(x)] d x=f(p) m(D)+\int_{D} a_{i j}^{\alpha \beta} \pi_{\alpha}^{i}(x) \pi_{\beta}^{j}(x) d x .
$$

But Van Hove [6] has shown that the condition (5.2) is necessary (this also follows from Theorem 4.3) and sufficient for the second integral to be $\geqq 0$ for all $\zeta$ of class $D^{\prime}$ on $D$ which vanish on $D^{*}$ (hence this is true also for all $\zeta$ of class $\Re_{2}$ on $D$ and vanishing on $D^{*}$ ).

LEMMA 5.2. Suppose

$$
\sum_{i, j=1}^{n} a_{i j} x^{i} y^{j}=0
$$

for all $x$ and $y$ for which

$$
\sum_{i, j=1}^{n} b_{i j} x^{i} y^{j}=0 .
$$

Then there is a constant $K$ such that 


$$
a_{i j}=K b_{i j}(i, j=1, \cdots, n) .
$$

Proof. We may introduce new variables $\xi$ and $\eta$ by

$$
x=c \xi, y=d \eta,
$$

$c$ and $d$ being $n \times n$ nonsingular matrices. Let $a$ and $b$ be the matrices of the original forms and $A$ and $B$ those of the transformed forms. Then

$$
A=c^{\prime} a d, \quad B=c^{\prime} b d \quad\left(c_{i j}^{\prime}=c_{j i}\right) .
$$

We shall show that there is a scalar $K$ such that $A=K B$. We may assume that

$$
B_{i i}=1 \quad(i=1, \ldots, r) ; \quad B_{i j}=0 \text { otherwise, } \quad r \leqq n,
$$

unless $B=0$ in which case $A=0$ also and the theorem holds. By taking $\eta^{s}=1$, $\eta^{j}=0 \quad(j \neq s, \mathbf{s}=1, \cdots, n)$ in turn we see that

$A_{i s}=0(i=1, \ldots, n, s>r) ; A_{i s}=0(i \neq s, s=1, \cdots, r, i=1, \cdots, n)$.

Then, by choosing $1 \leqq s<t \leqq r$ and setting $\eta^{s}=\eta^{t}=1, \eta^{j}=0, j \neq s, j \neq t$, we have

$$
\left(A_{i s}+A_{i t}\right) \xi^{i}=0 \quad \text { for all } \xi \text { with } \xi^{s}+\xi^{t}=0
$$

Thus there exists a constant $K(s, t)$ such that

$$
A_{s s}+A_{s t}=K(s, t), \quad A_{t s}+A_{t t}=K(s, t) .
$$

Hence

$$
A_{11}=A_{22}=\cdots=A_{r r}=K
$$

so that $A=K B$.

TheOREM 5.3. Suppose that $N=\nu+1$ and

$$
f(p)=F\left(X_{1}, \cdots, X_{\nu+1}\right),
$$

where $F$ is positively homogeneous of the first degree in the $X_{i}$ and

$$
\begin{aligned}
& X_{i}=-\operatorname{det} M_{i}(i=1, \cdots, \nu), X_{\nu+1}=\operatorname{det} M_{\nu+1}, \\
& M_{\nu+1}=\left\|p_{\alpha}^{1}, \cdots, p_{a}^{\nu}\right\|, M_{i}=\left\|p_{\alpha}^{1}, \cdots, p_{\alpha}^{i-1}, p_{\alpha}^{\nu+1}, p_{\alpha}^{i+1}, \cdots, p_{\alpha}^{\nu}\right\| \\
& \quad(i=1, \cdots, \nu) .
\end{aligned}
$$


Then $f$ is quasi-convex in $p$ if and only if $F$ is convex in the $X_{i}$.

Proof. If $F$ is convex in the $X_{i}$, it follows from Theorem 5.1 that $f$ is quasiconvex in $p$.

Hence suppose $f$ is given by (5.3) and is quasi-convex in $p$. If

$$
\Delta X_{k}=X_{k}\left(p_{a}^{i}+\lambda_{\alpha} \xi^{i}\right)-X_{k}\left(p_{\alpha}^{i}\right),
$$

then

$$
\Delta X_{k}=X_{k p_{a}^{i}} \lambda_{a} \xi^{i}
$$

Also, since

$$
p_{\beta}^{k} X_{k}=0 \quad(\beta=1, \cdots, \nu),
$$

we have

$$
p_{\beta}^{k} X_{k p_{\alpha}^{i}}=-\delta_{\beta}^{\alpha} X_{i}
$$

Now, choose a set of $X_{i}$ not all zero and choose any $p$ such that

$$
X_{i}(p)=X_{i}
$$

Since $f$ is quasi-convex and hence weakly so, there are constants $A_{\alpha}^{i}$ such that

$$
f\left(p_{\alpha}^{i}+\lambda_{\alpha} \xi^{i}\right) \geqq f(p)+A_{i}^{a} \lambda_{\alpha} \xi^{i}
$$

Since $f$ depends only on the $X_{i}$, we must have

$$
A_{i}^{\alpha} \lambda_{\alpha} \xi^{i} \leqq 0 \text { for all } \lambda, \xi \text { with } X_{k p_{\alpha}^{i}} \lambda_{\alpha} \xi^{i}=0(k=1, \cdots, \nu+1) .
$$

Obviously, then, the equality must hold in (5.6). Using (5.4) and (5.5), we see that

$$
p_{\beta}^{k} \Delta X_{k}=-\lambda_{\beta}\left(X_{i} \xi^{i}\right) \quad(\beta=1, \ldots, \nu) .
$$

Hence, we must have

$$
A_{i}^{a} \lambda_{\alpha} \xi^{i}=0
$$

for all $\lambda, \xi$ for which

$$
X_{i} \xi^{i}=0 \text { and } D_{i}^{\alpha} \lambda_{a} \xi^{i}=0, D_{i}^{\alpha}=X_{k} X_{k p_{a}^{i}} .
$$


Now, since not all the $X_{i}$ are zero, assume $X_{k} \neq 0$. Then

$$
\sum_{i \neq k}\left(A_{i}^{a} X_{k}-A_{k}^{a} X_{i}\right) \lambda_{a} \xi^{i}=0
$$

for all $\lambda, \xi$ for which

$$
\sum_{i \neq k}\left(D_{i}^{\alpha} X_{k}-D_{k}^{\alpha} X_{i}\right) \lambda_{a} \xi^{i}=0
$$

From the preceding lemma, it follows that there is a constant $K$ such that

$$
A_{i}^{\alpha} X_{k}-A_{k}^{\alpha} X_{i}=K\left(D_{i}^{\alpha} X_{k}-D_{k}^{\alpha} X_{i}\right) \text {. }
$$

Hence

$$
A_{i}^{\alpha}=K D_{i}^{\alpha}+L^{\alpha} X_{i}, \quad L^{\alpha}=X_{k}^{-1}\left(A_{k}^{\alpha}-K D_{k}^{\alpha}\right) .
$$

From (5.7) and (5.13) it follows that

$$
A_{i}^{\alpha} \lambda_{\alpha} \xi^{i}=K D_{i}^{\alpha} \lambda_{a} \xi^{i}+L^{\alpha} \lambda_{a} X_{i} \xi^{i}=C^{k} \Delta X_{k}, C^{k}=\left(K X_{k}-L^{a} p_{a}^{k}\right)
$$

Finally, if we are given any values of the $\Delta X_{k}$, the quantities

$$
h_{i}=p_{i}^{k} \Delta X_{k}(i=1, \cdots, \nu) \text { and } h_{\nu+1}=X_{k} \Delta X_{k}
$$

are determined and the $\Delta X_{k}$ are also uniquely determined by the $h_{i}$. Using (5.7), we may determine the $\lambda_{\alpha}$ in terms of the $h_{i}(i=1, \ldots, \nu)$, and substitute them into

$$
h_{\nu+1}=X_{k} \Delta X_{k}=D_{i}^{\alpha} \lambda_{\alpha} \xi^{i},
$$

and we merely have to choose the $\xi^{i}$ to satisfy the equation

$$
\left(D_{i}^{\alpha} h_{\alpha}+h_{\nu+1} X_{i}\right) \xi^{i}=0 \text { with } X_{i} \xi^{i} \neq 0 ;
$$

this is always possible unless all the $D_{i}^{\alpha} h_{\alpha}=0$. Thus, unless these linear relations in the $\Delta X_{i}$ hold, we have

$$
F(X+\Delta X)=f\left(p_{a}^{i}+\lambda_{a} \xi^{i}\right) \geqq f(p)+A_{i}^{\alpha} \lambda_{a} \xi^{i}=F(X)+C^{k} \Delta X_{k}
$$

The result follows in this case by continuity. 
Finally, since $F$ is homogeneous of the first degree, we see by taking

$$
\Delta X=h X, h>-1,
$$

that

$$
F[(1+h) X]=(1+h) F(X) \geqq F(X)+h C^{k} X_{k},
$$

or

$$
h\left[F(X)-C^{k} X_{k}\right] \geqq 0, h>-1 \text {. }
$$

Hence $F(X)=C^{k} X_{k}$. Then by setting $X=h X_{0}, X_{0} \neq 0$, choosing the $C^{k}$ for this $X_{0}$, and then letting $h \rightarrow 0$, we see that (5.15) holds for some $C^{k}$ even if $X=0$.

\section{REFERENCES}

1. J. W. Calkin, Functions of several variables and absolute continuity, I, Duke Math. J. 6 (1940), 170-186.

2. C. B. Morrey, Jr., Functions of several variables and absolute continuity, II, Duke Math. J. 6 (1940), 187-215.

3. Multiple integral problems in the calculus of variations and related topics, Univ. California Publ. Math., new series, 1, no. 1 (1943), 1-130.

4. The problem of Plateau on a Riemannian manifold, Ann. of Math. 49 (1948), 807-851.

5. F. J. Terpstra, Die Darstellung biquadratischer Formen als Summen von Quadraten mit Anwendung auf die Variationsrechnung, Math. Ann. 116 (1938), $166-180$.

6. L. Van Hove, Surl'extension de la condition de Legendre du Calcul des Variations aux integrals multiples a plusieurs fonctions inconnues, Nederl. Akad. Wetensch. 50, no. 1 (1947), 18-23.

University of California, Berkeley 



\section{EDITORS}

HERBERT BUSEMANN

University of Southern California

Los Angeles 7, California
R. M. ROBINSON

University of California

Berkeley 4, California

E. F. BECKENBACH, Managing Editor

University of California

Los Angeles 24, California

\section{ASSOCIATE EDITORS}

\author{
R. P. DILWORTH \\ HERBERT FEDERER \\ MARSHALL HALL
}

P. R. HALMOS

HEINZ HOPF

R. D. JAMES

\author{
BØRGE JESSEN \\ PAUL LÉVY \\ GEORGE PÓLYA
}

J. J. STOKER
E. G. STRAUS

KÖSAKU YOSIDA

\section{SPONSORS}

UNIVERSITY OF BRITISH COLUMBIA

CALIFORNLA INSTITUTE OF TECHNOLOGY

UNIVERSITY OF CALIFORNIA, BERKELLEY

UNIVERSITY OF CALIFORNIA, DAVIS

UNIVERSITY OF CALIFORNIA, LOS ANGELES

UNIVERSTTY OF CALIFORNIA, SANTA BARBARA

OREGON STATE COLLEGE

UNIVERSITY OF OREGON
UNIVERSETY OF SOUTHERN CALIFORNIA STANFORD UNIVERSITY

WASHINGTON STATE COLLEGE UNIVERSTTY OF WASHINGTON

AMERICAN MATHEMATICAL SOCIETY NATIONAL BUREAU OF STANDARDS, INSTITUTE FOR NUMERICAL ANALYSIS

Vari-Type Composition by

Elaine Barth

Delores Gilbertson

With the cooperation of

E. F. Beckenbach

E. G. Straus

Printed in the United States of America by

Edwards Brothers, Inc., Ann Arbor, Michigan

UNIVERSITY OF CALIFORNIA PRESS * BERKELEY AND LOS ANGELES

COPYRIGHT 1952 BY PACIFIC JOURNAL OF MATHEMATICS 


\section{Pacific Journal of Mathematics}

\section{Vol. 2, No. $1 \quad$ January, 1952}

Tom M. (Mike) Apostol, Theorems on generalized Dedekind sums ........ 1

Tom M. (Mike) Apostol, Addendum to 'On the Lerch zeta function' ........ 10

Richard Arens, Extension of functions on fully normal spaces ........... 11

John E. Maxfield, A short proof of Pillai's theorem on normal numbers.... 23

Charles B. Morrey, Quasi-convexity and the lower semicontinuity of multiple integrals................................ 25

P. M. Pu, Some inequalities in certain nonorientable Riemannian manifolds....................................... 55

Paul V. Reichelderfer, On the barycentric homomorphism in a singular

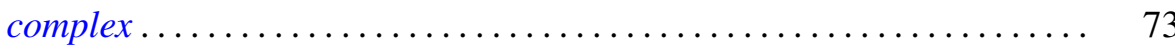

A. H. Stone, Incidence relations in multicoherent spaces. III ........... 99 